\title{
A Simulation Study on LTE Handover and the Impact of Cell Size
}

\author{
Muhammad Tayyab ${ }^{1(\bowtie)}$, George P. Koudouridis ${ }^{2}$, \\ and Xavier Gelabert ${ }^{2}$ \\ ${ }^{1}$ Huawei Technologies Finland Oy, Itämerenkatu 9, 00180 Helsinki, Finland \\ muhammad. tayyab5@huawei.com \\ ${ }^{2}$ Huawei Technologies Sweden AB, Skalholtsgatan 9, 16494 Kista, Sweden
}

\begin{abstract}
In this paper we address the impact of cell size on the handover procedure in a Long Term Evolution (LTE) network. In particular, we highlight the potential problems that may occur when small cell densification is applied. In addition, the impact of the User Equipment (UE) speed is also analyzed. System level simulations are provided using a detailed LTE network simulator accounting for multiple points-of-failure and channel modeling compliant with LTE standards. We conclude that a certain cell size can be found around which any increase or decrease of the cell size brings performance degradations due to different limitations in the uplink. The performance is also degraded as UE speed increases, especially for small cell sizes. And for large cell sizes, we note that low speed UEs handover failures may rise due to the inability to "escape" from a poor radio condition area.
\end{abstract}

Keywords: LTE $\cdot$ Handover $\cdot$ Performance evaluation $\cdot$ Simulation

\section{Introduction}

While the development of future cellular networks is often driven by the need for increased bit rates to support data-hungry applications, of equal importance is to provide reliable handover (HO) mechanisms as this directly impacts on the perceived quality of experience (QoE) for the end user. This is particularly true when, in order to provide such increased bit rates, we resort to the deployment of small cell networks in order to boost the capacity in a given area [1]. Cell densification has proven to be a spectral efficient method to increase capacity and has received large attention in recent years (see [2] and references therein). In addition to high data rate demands, networks will need to provide their services to users on the move, probably more so and at higher speeds with the appearance of new transport paradigms such as self-driving vehicles where the user, no longer at the steering wheel, may use that time for digital content consumption. With this in mind, in this paper we will address the impact of cell density and UE speed on the handover procedure in a Long Term Evolution (LTE) network.

While the study of the impact of cell size on the handover procedure is not new, the vast majority of the existing works are devoted to analyze the impact of small cell densification as opposed to a wider analysis covering a range of inter site distances (ISD). Moreover, a large number of works adopt a theoretical approach and, while interesting 
insights can be provided, there is sometimes a lack of fidelity with respect to the standard which causes that some effects are not appropriately captured. In particular the field of Stochastic Geometry has been in recent years used to model the impact of handover in dense small cell networks see for example [3-6]. Such works lack on capturing effects such as handover failures, and consequent re-establishment and cell-reselection procedures, Radio Link Control (RLC) failures and corresponding retransmissions, etc. In addition, a simulation-based approach is more appropriate to capture the details of the LTE standard. In [7], the performance of handover in dense small HetNets is discussed, i.e. considering an overlapping deployment of both macro and pico cells. In [8], authors examine and evaluate the impact of small cell deployments on mobility performance in LTE-systems. Analysis is done through system level simulations in various scenarios with macro and small cell overlaid deployments. In this paper we focus not only on the problems caused by higher densification but also the problems faced when the cell size increases. Detailed system level simulations considering a wide range of potential failure points are provided.

The rest of the paper is organized as follows: Sect. 2 provides an overview of the handover mechanism in LTE. Section 3 discusses the simulator implementation and modelling aspects. In Sect. 4, numerical results and discussion are given on the performance of LTE handover. Section 5 provides some concluding remarks.

\section{Handover Mechanism in LTE}

Handover $(\mathrm{HO})$ management is a relevant research point in cellular networks. The overall HO decision procedure in LTE is illustrated in Fig. 1 [9], and consists of four major steps. Firstly, the UE performs downlink (DL) signal strength measurements from both the serving eNBs and other neighboring eNBs. Within the context of HO the serving eNB is referred to as the source eNB or serving cell, while the neighboring eNBs are referred to as the target eNBs or target cell. In the second step these measurements are processed at the UE, and in the third step a measurement reports (measReport) is transferred to the serving eNB. In the fourth and final step, based on the measReport, the serving eNB makes the $\mathrm{HO}$ decision and sends a $\mathrm{HO}$ request message to the target eNB.

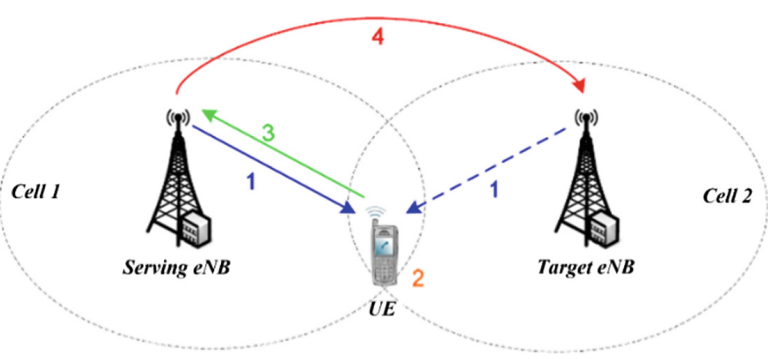

Fig. 1. HO process in 3GPP LTE [9]. 
The UE and eNB actions related to Radio Resource Management (RRM) procedures for the HO execution constitute the mechanisms for mobility control. In general, mobility control is divided into two stages, mobility control in RRC_IDLE state, which is referred to as cell reselection, and mobility control in RRC_CONNECTED state, which is referred to as handover. In this paper, we are focusing on mobility in RRC_CONNECTED state.

\subsection{Mobility Control in RRC_CONNECTED (Handover)}

There are two different types of actions that a UE has to perform in RRC_CONNECTED according to the requirements. The first type is, upon measuring and identifying a better neighboring cell than the serving cell, UE reports to the serving eNB and access the target cell on reception of HO command (from serving eNB). The UE performs signal strength measurements over specific reference signal received power (RSRP) from the serving cell as well as the neighboring cells. After processing the measurements, including filtering at layers L1 and L3, if an entry condition is fulfilled, a measurement report (measReport) is triggered to the serving cell. A3 event is used as entry condition to see if the filtered RSRP of the target cell is better than that of the serving cell plus a hysteresis margin (called A3 offset). The entry condition has to be maintained during a time defined by the Time to Trigger (TTT) timer. Once the measReport is correctly received at the serving cell, the HO preparation phase between target and serving cell starts which also includes admission control procedures. Upon successful admission, the target cell acknowledges the HO request sent by the serving cell and prepares for HO. Data forwarding starts between the serving and target cell and a $\mathrm{HO}$ command (HOcmd) is sent from the serving cell to the UE. Upon successful reception of the $\mathrm{HOcmd}$, the $\mathrm{HO}$ execution phase starts in which the UE accesses the target cell, by means of a random Access Channel (RACH) procedure, and delivers a HO confirmation (HOconf) message. Random access procedure takes two different forms, contention-based and contention-free RACH. In contention based RACH procedure, UE selects a random preamble and start transmission to the target eNB. But there is a chance of preamble collision due to the same preamble from multiple UEs. So, the network has to go through an additional process called contention resolution which is time consuming. In contention-free RACH procedure, the network informs each UE regarding when and which preamble it has to use. To initiate the contentionfree RACH process, UE should be in connected mode before the RACH process as in $\mathrm{HO}$ case. In order to achieve a good compromise between $\mathrm{HO}$ reliability and HO frequency, HO optimization deals with the adjustment of the TTT, A3 offset, and the L3 filter coefficient K [7].

The second type of action is the detection of the radio link failure (RLF) by monitoring the DL quality of the serving cell. Upon RLF detection, the UE selects the best target cell and gets its information for RRC connection re-establishment.

\section{Evaluation of Radio Link Failure (RLF)}

In RRC connected state, radio link monitoring enables the UE to determine whether it is in-sync or out-of-sync with respect to its serving cell. On getting consecutive number of out-of-sync indications, UE starts an RLF timer `T310' as shown in Fig. 2. Both in-sync 
and out-of-sync (N311 and N310) counters are configured by the network based on the associated in-sync threshold $\mathrm{Q}_{\text {in }}$ and out-of-sync threshold $\mathrm{Q}_{\text {out }}$ corresponding to $2 \%$ and $10 \%$ Block Error Rate (BLER), respectively. The timer stops when a number of consecutive in-sync (N311) indications are reported (case 2 in Fig. 2). If T310 expires, RLF occurs and UE turns off its transmission to avoid interference and try to re-establish a connection within a UE connection re-establishment delay (case 1 in Fig. 2). If the DL radio link quality becomes worse than Qout within a $200 \mathrm{~ms}$ period, then out-of-sync occurs. Whilst, in-sync occurs when the DL radio link quality becomes better than $\mathrm{Q}_{\text {in }}$ within a $100 \mathrm{~ms}$ period. These occurrences are reported by the UE to the physical layer and higher layers which may apply L3 filtering for evaluation of RLF [10].

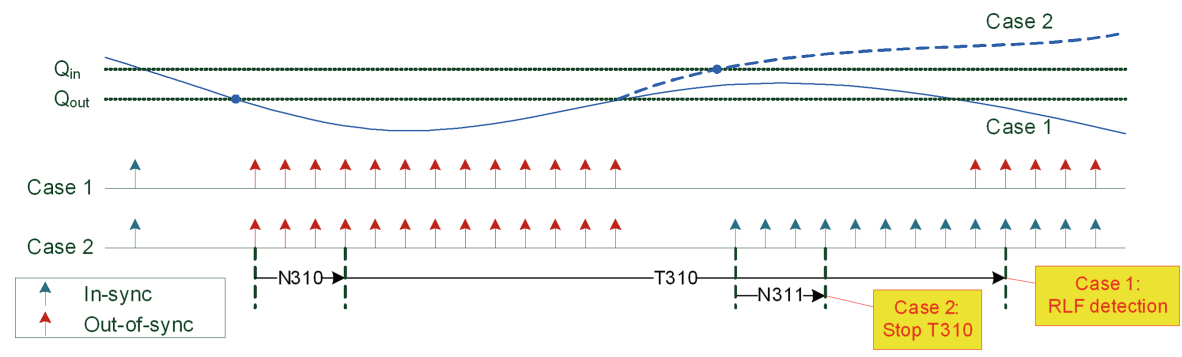

Fig. 2. RLF detection

An overall view of the HO procedure is shown in Fig. 3 [11], which can be divided into 3 phases: HO preparation (0-6), HO execution (7-11) and HO completion (12-14).

\section{System Simulation Models}

The used LTE simulator considers a hexagonal grid of 16 tri-sectored eNBs. Cell wraparound feature is included in order to ensure fair interference conditions across the scenario. UEs are randomly placed over the scenario and the mobility model is such that UEs move at fixed speed in straight lines with random directions $\left[0^{\circ}, 360^{\circ}\right]$.

As for traffic loading, UEs with UL full-buffer traffic are assumed, thus contributing to UL interference towards other UEs. DL interference is artificially generated by setting the transmission power on a number of randomly selected Physical Resource Blocks (PRBs) given a specific load level (in our case a fully loaded case is assumed).

The simulator implements the main features of the Packet Data Convergence Protocol (PDCP), Radio Link Control (RLC), Medium Access Control (MAC) and physical (PHY) layers including, inter alia, segmentation, Automatic Repeat Request (ARQ) at RLC level, and MAC scheduling with chase combined Hybrid-ARQ (HARQ). For the PHY layer, look-up tables are used which map bit error rate (BER) values to measured subcarrier Signal-to-Interference-and-noise-ratio (SINR) values (via the EESM, Effective Exponential SNR Mapping) in order to account for errors over the wireless link. 


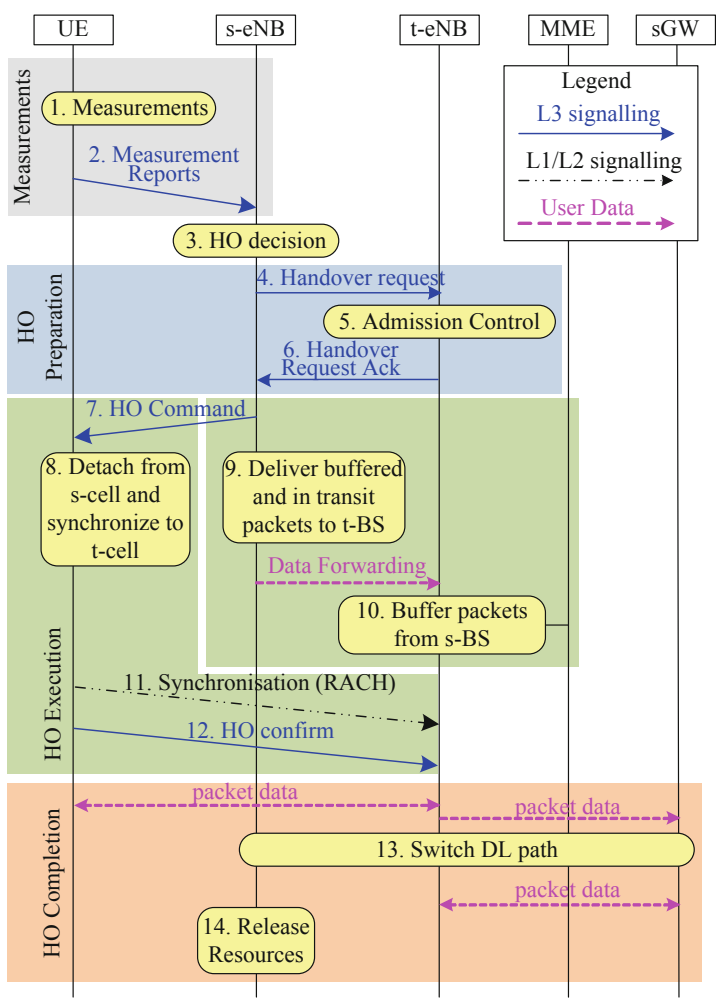

Fig. 3. Intra MME/SG HO procedure (adapted from [11]).

The HO model considers the modelling of L3 RRC signaling over the radio access, including measurement reports, handover command and handover confirmation. L2 signaling (UL and DL allocation) is also captured by modelling the PDCCH. Both L2 and L3 signaling are subject to channel impairments and thus prone to RLC failures.

Moreover, see Sect. 2.1, RLFs are also considered in the simulator with full modeling of the in-sync/out-of-sync states via counters N310, N311 and timer T310.

Summarizing, handover failures (HOF) are captured in the simulator falling into the following categories, with failure points being defined in Table 1.

1. RLF declared by L1 at the UE after timer T310 expiry [12, Sect. 7.3].

2. RLC is unable to deliver a Radio Resource Control (RRC) message after a (max.) number of transmission attempts. Applies to measReport and HOconf messages.

3. RACH failure after timer T304 expiry [12, Sect. 7.3].

The main simulation assumptions are summarized in Table 2. 
Table 1. Simulated failure points.

\begin{tabular}{l|l}
\hline Failure point & Description \\
\hline F0 & T310 expiry before measReport triggered \\
\hline F1 & T310 expiry before measReport received \\
\hline F2 & RLC measReport transmission error \\
\hline F3 & T310 expiry before HOcmd transmission \\
\hline F4 & T310 expiry before HOcmd reception \\
\hline F5 & RACH failure after T304 expiry \\
\hline F6 & T310 expiry before HOconf received \\
\hline F7 & RLC HOconf transmission error \\
\hline
\end{tabular}

Table 2. Simulation parameters and assumptions.

\begin{tabular}{l|l}
\hline Feature & Implementation \\
\hline Network topology & Hexagonal grid of $16 \times 3=48$ cells (wrap-around included) \\
\hline Inter-site distance & From the set $\{125,250,375,500,625,750,1000,1250\} \mathrm{m}$ \\
\hline Bandwidth & $5 \mathrm{MHz}$ FDD at $2.6 \mathrm{GHz}$ \\
\hline eNB DL power & $43 \mathrm{dBm}$ \\
\hline Antenna patterns & 3D model specified in [13], Table A.2.1.1.2-2 \\
\hline Channel model & 6 tap model, Typical Urban $(\mathrm{TU})$ \\
\hline Shadowing & Log-normal Shadowing Mean $0 \mathrm{~dB}$, Standard deviation: $8 \mathrm{~dB}$ \\
\hline Propagation model & $L=130.5+37.6 \log _{10}(R), R$ in $\mathrm{km}$ \\
\hline UE speed & From the set $\{3,30,60,120\} \mathrm{km} / \mathrm{h}$ \\
\hline RLF detection by L1 of UE & T310 $=1 \mathrm{~s}, \mathrm{~N} 310=1, \mathrm{~N} 311=1$ as specified in $[12]$ \\
& $\mathrm{Q}_{\text {in }}=-4.8 \mathrm{~dB} ; \mathrm{Q}_{\text {out }}=-7.2 \mathrm{~dB}$ as specified in [14] \\
\hline HO parameters & TTT $=64 \mathrm{~ms}, \mathrm{~A} 3$ offset $=3 \mathrm{~dB}, \mathrm{~L} 3$ filter coefficient $\mathrm{K}=4$ \\
\hline
\end{tabular}

\section{Performance Evaluation}

In this section we provide a numerical evaluation of the HO procedure under different conditions of cell density and UE speed. First, we start with some metric definitions.

\subsection{Metrics}

HO performance metric definition follows the guidelines in [15, Sect. 5.4.2]. The HO rate (HOR), measured in $\mathrm{HO}$ events/s, is defined as the total number of triggered $\mathrm{HO}$ events divided by the simulation time $(60 \mathrm{~s})$. Similarly, the HO failure ratio (HOFR), measured in $\%$, is defined as the total number of HO failure events divided by the total number of triggered $\mathrm{HO}$ events. The ping pong rate (PPR) is defined as the number of ping pong events during a given period of time. In turn, we define a ping pong event as the occurrence of a HO between a source cell and a target cell, followed by another HO to the original source cell, all this happening under a predefined time set to $3 \mathrm{~s}$. Finally, we define the mean time between handovers (MTBH) as the ratio between the total simulation time and the number of triggered $\mathrm{HO}$ events. 


\subsection{Numerical Evaluation}

Figure 4 illustrates the handover rate (measured in number of HOs per second) against the ISD and for different UE speeds. As expected, increasing the density of eNBs (lower ISD) results in an increase of HOs due to the increased number of cell borders. This rate notably increases when the ISD falls below $250 \mathrm{~m}$. Also expected is the increase of the HO rate with UE speed. In addition, an increase of the HO rate is also noted for increased ISD values, see ISD $=\{750,1000,1250\}$. The reason for this will become apparent when analyzing the different channel conditions impacting these deployments.

Next we drive our attention to the handover failure ratio (HFR), i.e. the percentage of those handovers which for one reason or another experienced problems and could not be completed seamlessly. Figure 5 illustrates the HFR against the ISD for different UE speeds. Both lowering and increasing the ISD have negative effects, i.e. increased HFR. For low ISD, failure arises due to adverse channel conditions due to excessive interference from neighbor UEs. Further insights on this will be provided later on. In addition, increasing UE speed contributes to HFR for lower ISDs (125 m, $250 \mathrm{~m}$ and $375 \mathrm{~m}$ ). We can argue that for small cells, higher UE speeds will cause moving away from the source cell which may cause problems during handover. For larger ISDs, $1 \mathrm{~km}$ and above, HFR also increases. This is again due to channel adversity as it will become apparent later on. In this case however, we note how the UE speed impact is reversed as compared to the low ISD case. For larger cells, "higher UE speed helps to escape from the cell border". Noteworthy, despite suffering handover failure, LTE implements re-establishment/cell-reselection mechanisms so as to recover the connection with the UE. However, this re-establishment comes at the cost of increased delay and interruption time.

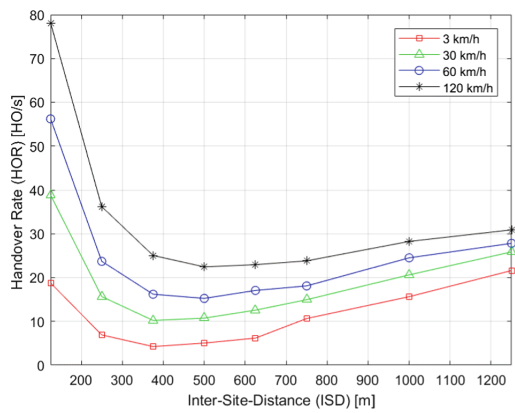

Fig. 4. Handover Rate (HOs/s) against the ISD for different speed values.

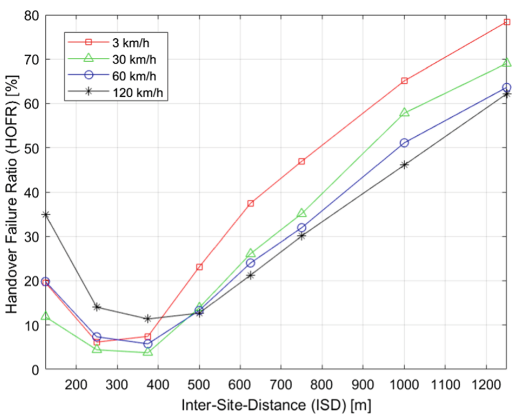

Fig. 5. Handover Failure Ratio (HOFR) in $\%$ against the ISD for different speed values.

Figure 6 shows the HO failure breakdown per type F0 to F7. An overall trend is that failures seem to concentrate on UL transmission errors (RLC timer/max. retransmissions expires). For high ISDs, a common failure is that of RACH failure which is 
due to the poor UL radio conditions for UEs close to cell borders in large cells. Notably at medium to high ISDs, higher failures are noted for lower speeds, indicating that the low speed is not enough to "escape" from these areas with poor conditions. F4 (T310 expiry before HOcmd reception) is very frequent when ISD is small and low when the ISD increases, whereas F5 (RACH failure after T304 expiry) is very frequent for large ISD and very low for small ISDs. The motivation is that, for small cell sizes, the UEs that are able to transmit the measurement report subsequently move out of the serving cell coverage and thus the HO command, sent by the serving cell, cannot reach the UE. For the RACH failures due to T304 expiry for large cells, the UEs transmitted power at the border of the cell compromises the success of the handover.

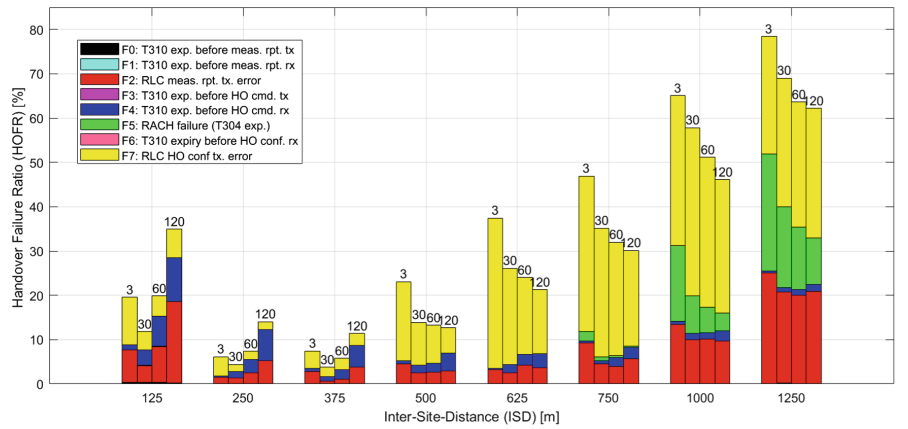

Fig. 6. Handover Failure Ratio (HOFR) in \% against the ISD for different speed values. HO failure type breakdown: F0...F7.

To find an explanation to the different HO failure cases we resort to the uplink interference and uplink signal-to-noise plus interference ratio. Below the uplink interference-over-thermal noise ratio and the uplink SINR are plotted in Figs. 7 and 8 respectively. As we see, low ISD (i.e. $125 \mathrm{~m}$ ) suffer from sever interference in the uplink since UEs are more likely to be closer to each other. However, these same UEs at lower ISDs suffer from better UL SINR conditions (see Fig. 8), since UEs are closer to the eNBs and thus the received power at those can partially compensate for the interference. On the contrary, deployments with larger ISD suffer less interference.

The ping pong rate is presented in Fig. 9. Ping pong events reflect the channel variability conditions, which cause HOs between two neighboring cells given successive A3 events being triggered. Ping pong events can also appear as the consequence of failed HOs to neighboring cells, and the subsequent efforts to reconnect the UE to neighboring cells even if not the most adequate at that time. Accordingly, both low and high ISDs seem to be prone to ping pong events due to channel quality impairments and subsequent HO failures (as already noted in Figs. 6, 7 and 8). An equivalent metric to the ping pong rate is that of the mean elapsed time between handovers $(\mathrm{MTBH})$ which is illustrated in Fig. 10. More frequent handovers (i.e. lower $\mathrm{MTBH}$ ) is noted for both low and high ISD values for the same reasons explained above. As expected, increasing UE speed results in both increased ping pong events and lower MTBH. 


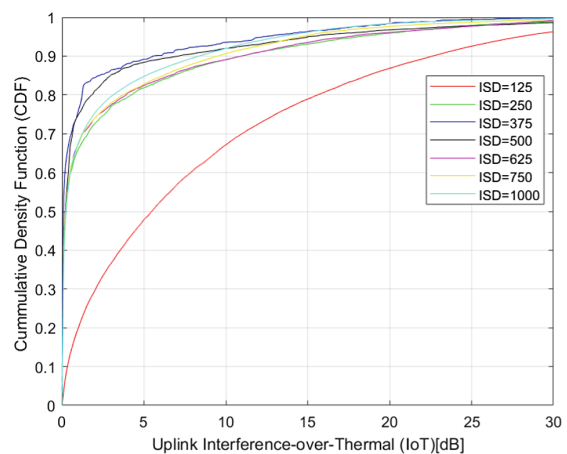

Fig. 7. UL interference-over-thermal noise ratio for different ISD values. UE speed is $30 \mathrm{~km} / \mathrm{h}$.

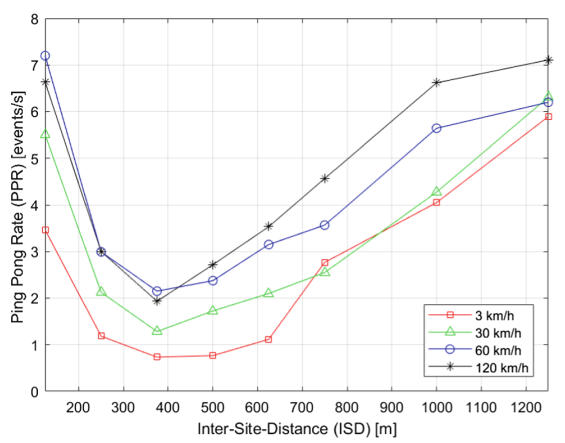

Fig. 9. Ping pong rate against ISD for different speed values.

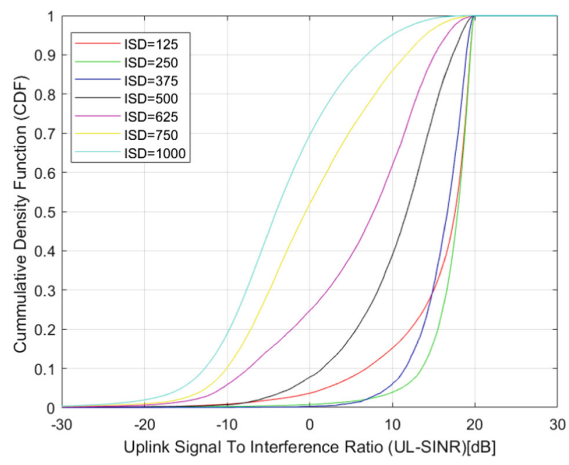

Fig. 8. UL signal to interference plus noise ratio (SINR) for different ISD values. UE speed is $30 \mathrm{~km} / \mathrm{h}$.

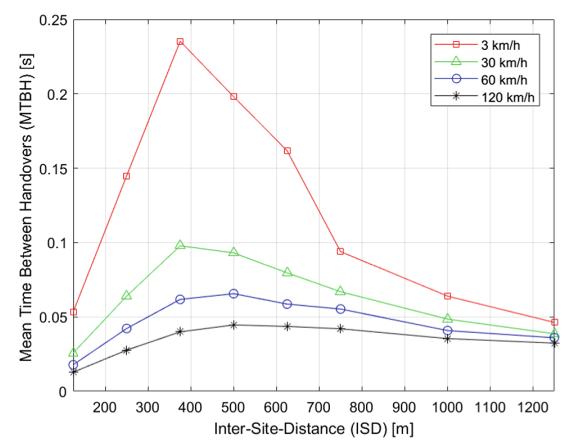

Fig. 10. Mean time between HOs (MTBH) against ISD for different speed values.

In the above, we have addressed the HO performance in terms of HOF and its causes. Noteworthy, LTE implements reestablishment and cell reselection mechanisms so as to recover from the suffered failures in the shortest time possible. Hence, noting that during part of the HO process, including the reestablishment procedure, the data plane will be unable to deliver packets to and from the UE, it seems reasonable to measure the elapsed time of this event. To this end, we define the HO interruption time (HOIT) as the time whereby the user plane is unable to deliver packets to and from the UE.

Figure 11 shows the empirical cumulative density function (CDF) for the HOIT for different UE speeds and fixed ISD of $250 \mathrm{~m}$. We observe how lower UE speeds benefit from lower HO interruption times, since fewer failures during the handover process are noted in this case (see e.g. Fig. 6). Note also how the HO interruption time is lower bounded thus preventing the curves to reach the $0 \mathrm{~ms}$ mark.

Next in Fig. 12 we show the empirical CDF of the HOIT for different ISDs and for a fixed UE speed of $30 \mathrm{~km} / \mathrm{h}$. Not surprisingly, HOIT increases both when the ISD is 


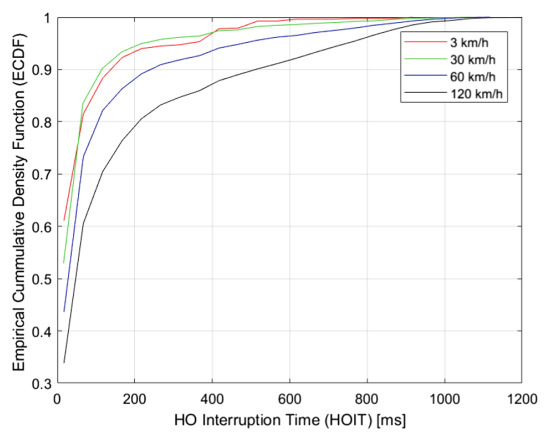

Fig. 11. Empirical cumulative density function $(\mathrm{CDF})$ of the $\mathrm{HO}$ interruption time for different UE speeds. ISD $=250 \mathrm{~m}$.

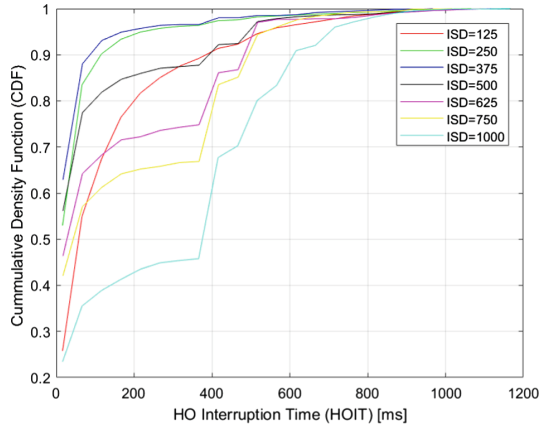

Fig. 12. Empirical cumulative density function $(\mathrm{CDF})$ of the $\mathrm{HO}$ interruption time for different ISDs. UE speed $=30 \mathrm{~km} / \mathrm{h}$.

small (ISD $=125 \mathrm{~m}$ ) and when it is larger (ISD = 1000). As mentioned earlier, both low and high ISDs involve different types of handover failures which in turn drive the MTBH to larger values in order to recover from such failures.

\section{Conclusions}

In this paper we have presented an in-depth simulation analysis on the causes for handover failure in an LTE network when different cell sizes are considered. In addition, the impact of the UE speed has also been assessed. Results show that a certain cell size can be found around which any increase or decrease of the cell size brings performance degradations due to different limitations in the uplink. For small cells, both the increased UL interference due to the proximity of neighboring UE and the higher rate of cell border crossings produce a severe handover degradation. For larger cells, the received power from the UE at the cell border is weak thus UL transmissions are impaired due to poor SINR. In addition, we also note a general degradation of the performance as UE speed increases, especially for small cell sizes. However, for large cell sizes we note that very low UE speeds handover failures may rise due to the inability to "escape" from a poor radio condition area. Future work will be devoted to analyzing the possible improvements via the optimization of the main handover parameters and by also reducing some failure points such as the measurement report transmission by having the network perform UE measurements and decide based on those whether or not to perform a handover.

Acknowledgments. This project has received funding from the European Union's H2020 research and innovation program under grant agreement H2020-MCSA-ITN- 2016-SECRET 722424. 


\section{References}

1. Gelabert, X., Legg, P., Qvarfordt, C.: Small cell densification requirements in high capacity future cellular networks. In: 2013 IEEE International Conference on Communications Workshops (ICC), pp. 1112-1116 (2013)

2. Anpalagan, A., Bennis, M., Vannithamby, R.: Design and Deployment of Small Cell Networks. Cambridge University Press, Cambridge (2015)

3. Arshad, R., et al.: Handover management in 5G and beyond: a topology aware skipping approach. IEEE Access 4, 9073 (2016)

4. Arshad, R., et al.: Handover management in dense cellular networks: a stochastic geometry approach. In: 2016 IEEE International Conference on Communications (ICC) (2016)

5. Lin, X., Ganti, R.K., Fleming, P.J., Andrews, J.G.: Towards understanding the fundamentals of mobility in cellular networks. IEEE Trans. Wirel. Commun. 12(4), 1686-1698 (2013)

6. Bao, W., Liang, B.: Stochastic geometric analysis of user mobility in heterogeneous wireless networks. IEEE J. Sel. Areas Commun. 33(10), 2212-2225 (2015)

7. Gelabert, X., Zhou, G., Legg, P.: Mobility performance and suitability of macro cell poweroff in LTE dense small cell HetNets. In: IEEE 18th International Workshop on Computer Aided Modeling and Design of Communication Links and Networks (CAMAD), pp. 99-103 (2013)

8. Yamamoto, T., Konishi, S.: Impact of small cell deployments on mobility performance in LTE-advanced systems. In: 2013 IEEE 24th International Symposium on Personal, Indoor and Mobile Radio Communications (PIMRC Workshops), pp. 189-193 (2013)

9. Ulvan, A., Bestak, R., Ulvan, M.: The study of handover procedure in LTE-based femtocell network. In: Third Joint IFIP IEEE Wireless and Mobile Networking Conference (WMNC), October 2010

10. Sesia, S., Toufik, I., Baker, M.: LTE the UMTS Long Term Evolution: From Theory to Practice, 2nd edn., pp. 503-529. Wiley, Chichester (2011)

11. 3GPP TS 36.300. (E-UTRA) and (E-UTRAN); Overall description; Stage 2 (Release 15), V15.0.0, Section 10, pp. 93-143, December 2017

12. 3GPP TS 36.331. E-UTRA Radio Resource Control (RRC); Protocol specification (Release 9), v9.2.0, March 2010

13. 3GPP TR 36.814. Further advancements for E-UTRA physical layer aspects (Release 9), V9.0.0, March 2011

14. 3GPP TS 36.133. Requirements for support of radio resource management (Release 9), v9.15.0, March 2013

15. 3GPP TR 36.839 V11.1.0. Mobility enhancements in heterogeneous networks (Release 11), December 2012 